\title{
Laparoscopic Cholecystectomy of Gangrenous Cholecystitis Safety and Feasibility
}

\author{
Sherif Abd-Al Fattah Saber, Ahmed Abd-Al Fattah Elshoura, Osama Hassan Abd-Raboh
}

General Surgery Department, Faculty of Medicine, Tanta University, Tanta, Egypt

\section{Email address:}

sherifabdelfattah@med.tanta.edu.eg (S. Abd-AlF. S.), sherifsaber1980@gmail.com (S. Abd-Al F. S.),

ahmed.elshoura@med.tanta.edu.eg (A. Abd-A1F.E.),dr_osama_hassan@med.tanta.edu.eg (O. H. Abd-Raboh)

\section{To cite this article:}

Sherif Abd-Al Fattah Saber, Ahmed Abd-Al Fattah Elshoura, Osama Hassan Abd-Raboh. Laparoscopic Cholecystectomy of Gangrenous Cholecystitis Safety and Feasibility. Advances in Surgical Sciences. Vol. 6, No. 1, 2018, pp. 16-19. doi: 10.11648/j.ass.20180601.13

Received: February 24, 2018; Accepted: March 11, 2018; Published: April 3, 2018

\begin{abstract}
Background: Laparoscopic Cholecystitis (LC) of gangrenous Cholecystitis (GC) which is a serious complication of acute Cholecystitis (AC) is challenging especially in the presence of dense inflammatory adhesions and pericholecystic collection. The aim of this study is to clarify the feasibility and safety of LC in GC. Materials and methods: This is a prospective study done in GIT and LAP surgery unit, General Surgery Department in Tanta university hospital between January 2014 and 2018 on 40 cases of GC from 350 cases of laparoscopymanaged AC. Demographic data, intraoperative time, difficulties of the procedure, postoperative ICU admission, and hospital stay and complications were recorded. Results: GC was suggested preoperatively from old age, DM, CVD, fever $>38^{\circ} \mathrm{C}$, tachycardia $>90$ beats /minute, leucocytosis $>13,000$ /cumm and gallbladder wall thickening $>4 \mathrm{~mm}$ and pericholecystic collection. LC introduces shorter time less difficulties intraoperatively, less need for ICU, shorter hospital stay and less frequent postoperative morbidity. Conclusion: The use of LC in GC is feasible and safe.
\end{abstract}

Keywords: Laparoscopic Cholecystectomy, Gangrenous Cholecystitis, Acute Cholecystitis

\section{Introduction}

$\mathrm{AC}$ is one of the most common reasons for emergency admission in surgical practice. Cholecystectomy is a gold standard treatment for AC [1].

$\mathrm{GC}$ is a serious complication of $\mathrm{AC}[1,2]$. It is the result of marked distension of the gallbladder (GB) causing increased tension in the bladder wall. Associated inflammation leads to ischemic necrosis of the wall, with or without associated cystic artery thrombosis [3].

Pre-operative diagnosis of this condition may prove difficult. It is more common in men and in patients with coexisting cardiovascular disease (CVD) and leukocytosis (white blood count $(\mathrm{WBC})$ greater than $17 \times 10^{3} / \mathrm{mm}^{3}$ ) [3] Other associated factors include diabetes (DM), critical illness and a high C-reactive protein level [4]. Once suspected, patients with GC generally undergo emergency Cholecystectomy in order to avoid life-threatening complications [3].

$\mathrm{LC}$ of $\mathrm{GC}$ is considered difficult when the procedure is prolonged and/or converted to open procedure. Significant factors which increased the operating time were: previous abdominal surgery, intrahepatic GB, multiple large calculi, thick walled GB, biliary anomalies and large distended GB. The reason for conversion was dense adhesions around the GB and in callot's triangle which made dissection extremely difficult. The low threshold for conversion to open surgery is a good sign for good and safe practice [5].

\section{Materials and Methods}

This is a prospective study done in GIT and LAP surgery unit, General Surgery Department in Tanta university hospital between January 2014 and 2018 on 40 cases of GC from 350 cases of laparoscopy managed AC. The decision for patient inclusion was taken intraoperatively after diagnosis of gangrenous cases. The study was approved by the ethical committee in our hospital and all patients had written consent before enrollment in the study.

The diagnosis of all cases of AC depended on preoperative history taking, clinical examination, pelvi-abdominal 
ultrasound, laboratory investigations including liver function tests (ALT, AST, Total and direct bilirubin, alkaline phosphates, GGT and serum amylase), kidney function tests, complete blood picture and coagulation profile (PT\&PTT).

Operatively, LC was performed by the four-port technique. Briefly, carbon dioxide was used for peritoneal insufflations, and abdominal pressure was maintained between 10 and 14 $\mathrm{mm} \mathrm{Hg}$. GBs were covered by adherent omentum. The omental adhesions were gently freed revealing either patchy or diffuse gangrenous GB, which was thick, black and distended. After puncture and evacuation of GB content it was caught by Endoclinsh or Grasper forceps in the area of Hartman pouchand it is conducted a traction upwards and lateral. Then with the use of Hook connected by monopolar diathermy dissection of the medial GB wall was performed, then exploration of cystic duct and identification of its fusion with common hepatic duct was done and lateral wall of GB is separated to create the critical view of safety. Cystic duct was dissected, clipped or ligated and intersected, but in some cases we had observed the necrosis of GB wall extends into Hartman pouch and distal part of cystic duct. In such cases it is necessary to separate a healthy area of cystic duct and its further clipping. Cystic artery was also prepared, clipped and intersected and Then GB was separated from the liver and extracted out through epigastric port. Finally, peritoneal lavage using normal saline and drainage was routinely applied [Figure 1 and 2].

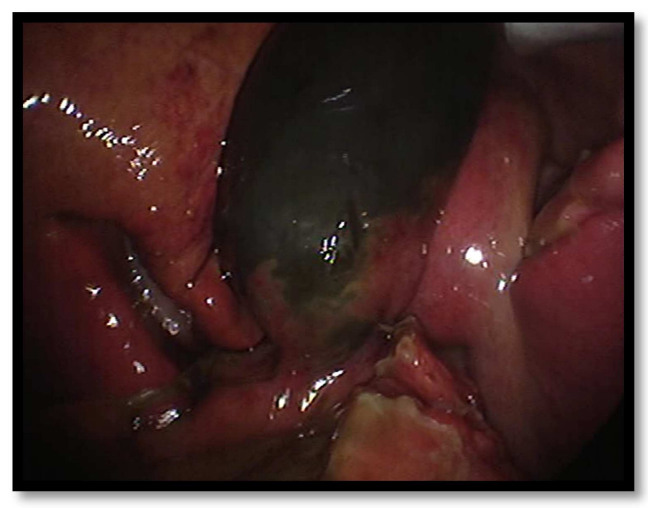

Figure 1. Diffuse gangrenous Cholecystitis.

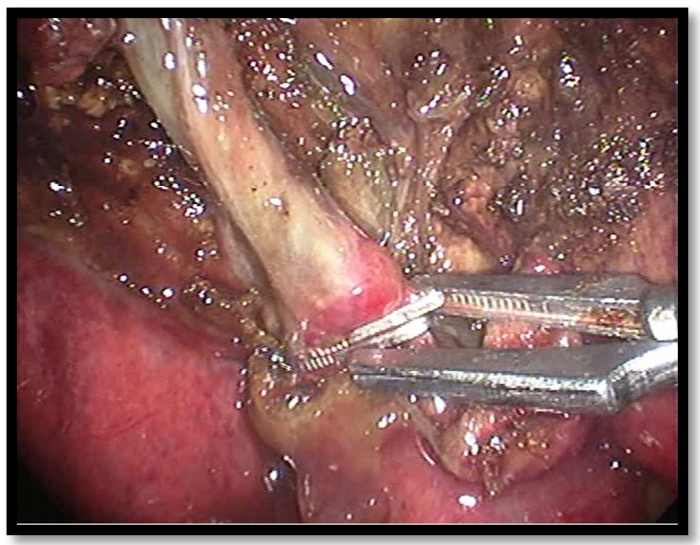

Figure 2. Clipping of the healthy proximal part of cystic duct.
If there was an evident inflammatory process, dense infiltrate in the given anatomic area that couldn't be dissected laporascopically, a laparotomy should be used to avoid the prolongation of operative time and accidental injury of the involved organs. Pathological diagnosis of the extracted GB was routinely performed.

Intraoperative time, difficulties of the procedure, postoperative ICU admission, and hospital stay and complications were recorded.

\section{Results}

\subsection{Pre-Operative Data [Table 1]}

A clinical picture of persistent right hypochondrium and epigastric pain with temperature more than 37.5 , tenderness and guarding rigidity of upper abdomen, leukocytic count more $10,000 / \mathrm{mm}^{3}$, in addition to positive Murphy's sign on ultrasound or the presence of GB wall edema and pericholecystic collection are diagnostic of AC.

Only 13 cases of GC were suggested preoperatively of having gangrenous changes from old age, DM, CVD, fever $>$ $38^{\circ} \mathrm{C}$, tachycardia $>90$ beats /minute, leukocytosis $>13,000$ $/ \mathrm{mm}^{3}$ and GB wall thickening $>4 \mathrm{~mm}$ and pericholecystic collection.

Table 1. Demographic data and preoperative risk factors.

\begin{tabular}{ll}
\hline Preoperative risk factors & GC $(\mathbf{n}=\mathbf{4 0})$ \\
\hline Age: & 61,3 ys (mean) \\
Sex (male:female) & $1.4: 1$ \\
D. M: & $12 / 40(30 \%)$ \\
CVD: & $15 / 40(37.5 \%)$ \\
Temperature: & $38,2^{\circ} \mathrm{C}($ mean $)$ \\
Tachycardia: & $98 \mathrm{~b} /$ min (mean) \\
Leukocytosis: & $18,300 / \mathrm{mm}^{3}($ mean $)$ \\
GB thickness (US) & $4,6( \pm 1.7) \mathrm{mm}$ \\
\hline
\end{tabular}

\subsection{Operative Data [Table 2]}

The diagnosis of GC was based on operative findings and detection of full-thickness necrotic areas along with infiltration by neutrophils and mononuclear cells in histologic sections. The gangrene of GB was defined as focal if one portion of the GB wall was involved and as diffuse if more than one portion was involved. Gangrenous-perforative form was determined in $(3 \%)$ patients. Different forms of peritonitis were revealed. Peritonitis was either local (transsudative) or diffuse. The diffuse exudates were serous, serous-bilious, purulent or fibrinous exudates.

Difficult LC was defined as procedure exceeding 80 minutes in duration and / or converted to open procedure. 34 cases of GC completed laporascopically while the other 6 Patients underwent conversion to the open procedure due to difficult exploration ( 3 cases), bleeding ( 2 cases) and bile duct injury (one case). 
Table 2. Demonstrates the course of the laparoscopy.

\begin{tabular}{ll}
\hline Intraoperative factors & GC \\
\hline Operative time & $78 \pm 11$ minutes \\
Difficulties: & \\
Easy laparoscopy & $22 / 40(55 \%)$ \\
Prolonged laparoscopy with difficulties & $12 / 40(30 \%)$ \\
Conversion to open & $6 / 40(15 \%)$ \\
\hline
\end{tabular}

\subsection{Post-Operative Data [Table 3]}

5 cases required ICU admission 3 of them had intraoperative difficulties with conversion to open while the other 2 cases however they had easy laparoscopic course but they required admission owing to uncontrolled CVD. While the postoperative hospital stay was variable as short as 2 days in the uncomplicated cases who represented the majority of our study and as long as 12 days in complicated cases with mean $3.0 \pm 1.2$ days. The overall postoperative complications were in 4 cases $(10 \%)$ distributed between 2 cases of them had Subhepatic collection and port site infection, one had biliary leakage through the drain andport site infection and one other case had biliary leakage.

Table 3. Demonstrates the postoperative complications.

\begin{tabular}{ll}
\hline Postoperative finding & No. of GC \\
\hline Hospital stay & $2-12$ days (mean $3.0 \pm 1.2 \mathrm{ds})$ \\
Admission to ICU & $5 / 40(12.5 \%)$ \\
Postoperative complications: & \\
overall morbidity & 4 cases $(10 \%)$ \\
1. Subhepatic collection & 2 cases $(5 \%)$ \\
2. Port site infection & 3 cases $(7.5 \%)$ \\
3. Biliary leak through the drain & 2 cases $(5 \%)$ \\
\hline
\end{tabular}

\section{Discussion}

GC develops in $2 \%$ to $20 \%$ of the cases with acute Cholecystitis [5, 6, 14]. According to our data this form of $\mathrm{AC}$ has been revealed in $11.4 \%$ patients. Epithelial injury by increasing GB wall tension is owing to vascular insufficiency arising secondary to persistent obstruction of the cystic duct [5]. In addition to the phospholipases released from cell membranes of damaged epithelium initiate heavy inflammatory reaction $[1,2,5,15]$. Inflammation and ischemia of the gallbladder wall show progressive worsening as a result of deteriorating venous insufficiency with age, thereby giving rise to more necrosis and perforation $[7,16$, 17]. The risk of developing GC is higher in males compared with females [14]. In the current study, mean age incidence was 61.3 years and male to female incidence was 1.4: 1 .

A randomized controlled trial (RCT) to specify the risk factors on mortality in GC by Akın "Onder reported 24.3\% of cases ofGC had CVD and $19.6 \%$ had DM [12]. In this study the co-morbidity presented in $57.5 \%$ in all cases mainly CVD $(37.5 \%)$ and DM (30\%). It has been proposed that pathologies of the cystic artery emerging from atherosclerosis or microvascular diseases might lead to vascular insufficiency, therefore predisposing factors such as DM and CVD could be regarded as risk factors in development of GC [13].

A high degree of leukocytosis has been documented to be correlated with the infection severity in the GB wall [17]. Aydin et al [18] reported that WBC count $15,000 / \mathrm{mm}^{3}$ predicted development of $\mathrm{GC}$, whereas it was $17,000 / \mathrm{mm}^{3}$ in the study by Merriam et al [19]. The number of WBCs in the patients with GC in this study was detected to be high with mean $18,300 / \mathrm{mm}^{3}$.

The hallmark on sonography of GC is the presence of heterogeneous or striated thickening of the GB wall, which is often irregular with projections into the lumen and pericholecystic fluid collections [15]. The presence of intraluminal membranes representing desquamative GB mucosa is a specific finding but it is less common [3]. The accuracy of pre-operative ultrasound in diagnosing $\mathrm{GC}$ remains uncertain. The ultrasound study in our cases played role in predicting gangrenous changes in some cases preoperatively by increased thickness of the wall of GB with mean 4,6 ( \pm 1.7) $\mathrm{mm}$, pericholecystic collection and intracystic air in some cases.

Due to non-specific clinical and laboratory findings the diagnostics of GC remains a dilemma for operating surgeons. GC in old-aged patients is diagnosed with difficulty thereof such patients have an erased clinical picture of disease. Degree of morphologic changes in GB does not often correspond to clinical presentations of disease, especially to the intensity of pains in abdomen [7]. And this explains why only 13 cases of GC were suggested preoperatively of having gangrenous changes in our study.

KHADJIBAEV AB. et al in study of acute GC, LC recorded average Intraoperative time 75.0 17.33 minutes while in conventional Cholecystectomy it was $85.0 \pm 20.00$ minutes [20]. In this current study the average Intraoperative time was $78 \pm 11$ minutes so we find that shorter time is required with LC than open Cholecystectomy.

In study conducted by Sahu SK. et al [6] Out of 200 LC, $130(65 \%)$ were easy and $70(35 \%)$ were considered as difficult. Out of these 70 difficult cases $12(6 \%)$ required conversion to open Cholecystectomy, while in this study the results were $55 \%$ of cases were easy, $30 \%$ of cases had prolonged laparoscopy with difficulties and $15 \%$ of cases were converted to open, several factors played role in difficult laparoscopy varying between previous abdominal surgery, intrahepatic GB, multiple large calculi, thick walled GB, biliary anomalies, large distended GB, dense adhesions around the GB and in callot's triangle, bleeding and bile duct injury.

Conversion to open surgery was dictated by difficulties in dissection due to adhesions, insufficient anatomic exploration, complications like bleeding and injury to bile ducts, and technical difficulties [13]. The literature reports conversion rates between $30 \%$ and $75 \%$ [9]. Most reports have cited improved conversion rates as they gain experience in laparoscopic surgery [11] and the development of several surgical instruments used for laparoscopic procedures may also have contributed to decrease in the conversion rate and better control of Intraoperative bleeding [8]. A RCT of laparoscopic versus open cholecystectomy for acute and GC by Kiviluoto reported $16 \%$ patients in the LC group requiring conversion and in most cases because severe inflammation 
distorting the anatomy of Callot's triangle [10] and this study records near result of $15 \%$ conversion to open Cholecystectomy due to difficult exploration (3 cases), bleeding ( 2 cases) and bile duct injury (one case).

In the current study $5 \%$ of patients were converted to open due to bleeding while this percentage was $9 \%$ due to bleeding that could not be controlled adequately in the study conducted by Stefanidis D et al on 238 patients [16].

In this study 5 cases $(12.5 \%)$ required ICU admission, 3 of them had Intraoperative difficulties with conversion to open while the other 2 cases however they had easy laparoscopic course but they required admission owing to uncontrolled CVD. The ICU admission was $9.2 \%$ of the patient of laporascopic group but $82.6 \%$ patients in open cholecystectomy group need to be stayed in ICU in ABDUKHAKIM KHADJIBAEV et al study [20], this indicates lesser requirement for ICU admission with LC.

While the postoperative hospital stay was variable as short as 2 days in the uncomplicated cases who represented the majority of our study and as long as 12 days in complicated cases with mean $3.0 \pm 1.2$ days. The hospital stay was ranging $1-28$ (mean8.0 \pm 5.7 ) days in study by Ak1n "Onder [12] and the hospital stay was $14.0 \pm 2.03$ days in laparoscopic group versus $36.0 \pm 4.99$ days in open group in study conducted by ABDUKHAKIM KHADJIBAEV [20].

The overall postoperative complications were in 4 cases (10\%) which falls within complication rates of $9-20 \%$ reported by other studies [21], which is much lesser than that reported with open laparoscopy by other study where it was $20.5 \%$ [16], the complications were distributed between 2 cases of them had Subhepatic collection and port site infection, one had biliary leakage through the drain andport site infection and one other case had biliary leakage.

\section{Conclusion}

GC is associated with increased morbidity and mortality compared with that of AC. LC approach is a safe procedure for patients with GC and is associated with decreased morbidity. LC should be used when possible for GC to minimize postoperative complications however Open Cholecystectomy should not be avoided if necessary to ensure patient safety.

\section{References}

[1] Yamashita Y, Takada T \& Hirata K (2006) A survey of the timing and approach to the surgical management of patients with acute cholecystitis in Japanese hospitals. J Hepatobiliary Pancreat Surg; 13: 409-415.

[2] Beldi G, Glattli A (2003) Laparoscopic subtotal cholecystectomy for severe cholecystitis. SurgEndosc; 17:1437-9.

[3] Shinke G, Noda T \&Hatano H (2015) Feasibility and Safety of Urgent Laparoscopic Cholecystectomy for Acute Cholecystitis After 4 Days from Symptoms Onset. J Gastrointest Surg; 19:1787-1793.
[4] Bennett GL, Rusinek H, Lisi V, et al (2002) CT findings in acute gangrenous cholecystitis. AJR Am J Roentgenol; 178 (2):275-281.

[5] Sahu S, Agrawal S \& Sachan P (2003) Intraoperative difficulties in laparoscopic cholecystomy. Jurnalul de Chirurgie (Iaşi); 9: 2-5.

[6] ContiniS, Corradi D, Busi N, et al (2004) Can gangrenous cholecystitis be prevented?: a plea against a 'wait and see' attitude. J Clin Gastroenterol; 38 (8):710-716.

[7] Ukhanov A, Baydo S, Chakhmachev S, et al (2009) "The advantages of laparoscopic cholecystectomy in old patients with acute calculous cholecystitis," Abstracts of the 12th Russian congress of endoscopic surgeons, Endoscopic surgery. JurnalEndoskopicheskayaHirugiya], in Russian; 1: 45 - 51.

[8] Shinke G, Noda T, Hatano H, et al (2015) Feasibility and Safety of Urgent Laparoscopic Cholecystectomy for Acute Cholecystitis after 4 Days from Symptom Onset. J Gastrointest Surg; 19:1787-1793.

[9] Singer J \&Mckeen R (1994) Laparoscopic cholecystectomy for acute or gangrenous cholecystitis. Am. J. Surg; 60: 326-8.

[10] Kiviluoto T, Sinen J \& Lwkkanen P (1998) Randomized trial of laparoscopic versus open cholecystectomy for acute and gangrenous cholecystitis. The Lancet; 351: 321-325.

[11] Hunt D \& Chu F (2000) Gangrenous cholecystitis in the laparoscopic era. Aust. N. Z. J. Surg; 70: 428-430.

[12] Onder A, Kapan M, "Ulger B, et al (2015) Gangrenous Cholecystitis: Mortality and Risk Factors. IntSurg; 100:254-260.

[13] Fagan S, Awad S, Rahwan K, et al (2003) Prognostic factors for the development of gangrenous cholecystitis. Am J Surg; $186(5): 481-485$.

[14] Aydın C, Altaca G, Berber I, et al (2006) Prognostic parameters for the prediction of acute gangrenous cholecystitis. J HepatobiliaryPancreatSurg; 13 (2):155-559.

[15] Bennett G, Rusinek H, Lisi V, et al (2002) CT findings in acute gangrenous cholecystitis. AJR Am J Roentgenol; 178 (2):275-281.

[16] Stefanidis D, Bingener J, Richards M, et al (2005) Gangrenous Cholecystitis in the Decade Before and After the Introduction of Laparoscopic Cholecystectomy. JSLS; 9:169173.

[17] Schafer M, Krahenbuhl L \& Buchler M (2001) Predictive factors for the type of surgery in acute cholecystitis. Am J Surg; 182 (3):291-297.

[18] Aydin C, Altaca G, Berber I, et al (2006) Prognostic parameters for the prediction of acute gangrenous cholecystitis. J Hepatobiliary Pancreat Surg; 13 (2):155-559.

[19] Merriam L, Kanan S, Dawes L, et al (1999) Gangrenous cholecystitis: analysis of risk factors and experience with laparoscopic cholecystectomy. Surgery; 126 (4):680-685.

[20] Khadjibaev A, Tajanov S, Khadjibaev F, et al (2011) Laparoscopic Cholecystectomy in acute gangrenous Cholecystitis. MHSJ; 5: 43-48.

[21] Wevers K, van Westreenen H\& PatijnG (2013) Laparoscopic cholecystectomy in acute cholecystitis: C-reactive protein level combined with age predicts conversion. Surg LaparoscEndoscPercutan Tech; 23:163-6. 